ISSN 2766-5879

\title{
Absorbable Hemostatic Particles to Reduce the Damage on the Follicular Reserve After Ovarian Endometriomas Stripping
}

\author{
Gynecology and Women's Health Care
}

Research Article

\author{
Bardi M*, Pezzetti G, Laquintana R, Chiesa A, Gangarossa G, Iliakis A, Salerno B, Rebecchi A and Rosaschino P \\ *Correspondence author \\ Department of Obstetrics and Gynecology - Polyclinic San \\ Massimo Bardi \\ Pietro (San Donato Group), Ponte San Pietro (BG) - Italy \\ Senior Consultant of Gynecology in Polyclinic San Pietro \\ Department of Obstetrics and Gynecology \\ Italy
}

Submitted : 16 Nov 2021 ; Published : 30 Nov 2021

\begin{abstract}
Objective: to reduce the surgical damage to the ovarian reserve, after stripping of ovarian endometrioma, of the necrotic type given by the electrohaemostasis or ischemic type given by the suture.

Design: perform haemostasis on ovarian parenchyma with topical haemostatic agents.

Materials and methods: we used Arista AH which is a powder made up of microporous polysaccharide hemospheres that act by osmotic action and accelerate the natural coagulation process. We used Arista AH in 27 women with mono- or bilateral ovarian endometriosis.

Results: in all treated cases we obtained a rapid and optimal haemostasis. There were no post-surgical complications related to haemostatic defects. Three months after the surgery, we checked the Antral Follicle Count (AFC) with a trans-vaginal ultrasound probe. AFC in 24 women with full follow-up gave the following results:

- unilateral endometrioma - AFC between 5 and 8 (MV: 6.3),

- bilateral endometriomas - the AFC between 5 and 7 (MV: 5.8).

Conclusions: The use of Arista AH powder allows highly effective hemostasis and is easy to use, fully respecting the residual ovarian parenchyma after stripping.
\end{abstract}

Keywords: Ovarian Endometriosis, Follicular Reserve, Laparoscopic Surgery, Hemostasis

\section{Introduction}

Endometriotic ovarian cyst (endometrioma) is one of the three clinical forms in which endometriotic disease occurs. The endometriotic pathology is characterized by the presence of a like-uterine endometrium that is located outside of the uterus and functionally responds to ovarian hormonal stimuli. Endometriosis is estimated to affect around $10 \%$ of women in their reproductive years [1]. Although a causal relationship between endometriosis and infertility has not been demonstrated, subfertility is present in $30-50 \%$ of patients with endometriosis. The disease can affect fertility by inducing an inflammatory reaction in the pelvic area, altering tuba-ovarian function and endometrial receptivity [2].

Endometriosis and ovary

Ovarian endometriomas are present in $17 \%$ to $44 \%$ of women with endometriosis [3]. The endometrioma transforms the macro- and microenvironment in the ovary to a highly inflammatory one. The endometriotic tissue may secrete any inflammatory products that can activate specific pathways in the follicular cells, until a premature follicular development and accelerated atresia [4]. The Donnez group had shown a significant reduction in the number of primitive follicles in the cortical stroma adjacent to the endometrioma and, after two years, the same group formulated the "burnout hypothesis" [5]. The formation of endometrioma can cause inflammation and fibrosis in the ovarian cortex which causes focal loss of follicular density along with dysregulated folliculogenesis which results in "depletion" of the reserve of dormant follicles [6]. Nicolaus and Coll. determined the influence of endometriosis on the ovarian response during ovarian hyperstimulation as measured by the number of oocytes retrieved and the follicular output rate (FORT). They demonstrated that endometriosis reduces FORT and the number of oocytes in metaphase-II regardless of women's age, antral follicle count and anti-Müllerian hormone [7].

From this data it is clear that endometrioma can damage the follicular reserve. Even if many authors disagree [8,9].

\section{Ovarian endometriosis and surgery}

Among the causes through which endometriosis causes infertility we must not forget the iatrogenic damage resulting 
from inadequate surgery. Roman and Coll. have observed that endometrioma cystectomy, despite careful surgical technique, leads to frequent removal of ovarian tissue and the amount of ovarian parenchyma loss increases proportionally as the diameter of the cyst increases. This tissue loss averages $200 \mu \mathrm{m}$ per centimeter of increased cyst diameter [10]. For this reason it is necessary to improve the surgical technique of ovarian endometrioma. It is necessary to evaluate the benefits that surgery can obtain in an attempt to optimize fertility compared to the damage it can cause. Furthermore, the surgery must be performed by experienced surgeons who use a meticulous technique aimed at avoiding follicular damage, preventing post-surgical training and re-formation of adhesions [11].

Let us remember how shareable what Hurt et Coll. conclude in a 2008 Cochrane review, "There is good evidence that excisional surgery for endometrioma provides a more favorable outcome than drainage and ablation, with respect to endometrioma recurrence. endometrioma, the recurrence of pain symptoms and the next spontaneous pregnancy in women who were previously subfertile "[12].

\section{The "hemostasis" problem}

The stripping technique frequently causes bleeding, sometimes profuse, in the dissection area. The problem of hemostasis therefore arises and its resolution is often difficult. Bipolar coagulation is the most widely used method and, however practical and effective, it can cause further destruction of ovarian tissue, causing irreversible thermal damage to the follicular reserve, indicated by low levels of post-surgical $\mathrm{AMH}$ and AFC $[13,14]$.

Many authors have tried to compensate by using the laparoscopic suture as a hemostatic method. This technique involves approaching the ovarian and medullary cortex, using a suture with absorbable threads. At a 3-month follow-up, sutured patients had higher AMH and lower FSH (statistically significant: $\mathrm{p}<0.001$ ) than patients in whom electrocoagulation was used. However, some secondary effects must also be considered such as the duration of the surgery which is longer when suturing is performed resulting in more blood loss [15]. Mahmoud reports of a patient who developed ovarian hematoma after suturing [16]. Takashima and Coll. argue that laparoscopic suture can have a more detrimental effect on ovarian reserve than electrocoagulation because it can ischemize the cortical tissue of the ovary resulting in follicular damage [17].

Topical haemostatic agents could be a valid alternative to reduce damage due to other haemostatic techniques (devascularization and tissue necrosis). Hemostatic agents achieve effective hemostasis, reduce blood loss, reduce the risk of perioperative transfusions, and shorten surgical times [18]. A systematic review of the use of a hemostatic agent after removal of endometriomas indicated that the method better preserves serum AMH levels than bipolar energy. This experience was confirmed by subsequent studies that examined thrombin / gelatin matrix or fibrin sealant for hemostasis showing less reduction in AMH than bipolar energy consumption assessed one month after surgery [19].

\section{Our experience}

In relation to a previous experience with hemostatic agents that has provided good results in terms of respect for the ovarian reserve we decided to use Arista $\mathrm{AH}$ powder for haemostasis in all patients with ovarian endometriosis to undergo surgery [20]. Arista AH (Absorbable Hemostat) consists of microporous polysaccharide hemospheres with a controlled pore size. Hemospheres act by osmotic action and, once in contact with blood, dehydrate it, concentrating platelets, red blood cells and proteins to form a gelled matrix. This accelerates the natural coagulation process, regardless of the patient's state of coagulation. Arista AH is then absorbed within 24-48 hours of application.

Only women with symptoms resistant to medical therapy, women with associated other pathologies (DIE, myomas, etc.) and women with diagnosed infertility, after at least 6 months of unsuccessful attempts, were admitted to surgery according to the protocol indicated in table no. 1

Patients with documented ultrasound findings of mono- or bilateral ovarian endometrioma with diameter $=>4 \mathrm{~cm}$.

Clinical evaluation with vaginal and / or rectal exploration Transvaginal Ultrasound for the study of:

- Morphology and volume of both ovaries endometrioma measurement with 3 diameters

- Myometrial ecostructure and endometrial pattern

- Rectovaginal septum - Douglas pouch with sliding sign

- Bladder-uterine septum with sliding sign

In case of endometrioma fixation on the pelvic wall: renal ultrasound.

In case of suspected multifocality of endometriosis or associated DIE: Nuclear Magnetic Resonance

Table 1: Admission protocol to surgery for ovarian endometrioma

\section{Our surgical procedure}

After placing the bladder catheter and uterine manipulator, four trocars were inserted as in classic laparoscopic gynecological surgery. We first performed the lysis of the loosened pelvic adhesions for histological evaluation. Then, we open and drain the endometrioma (which often ruptures spontaneously during mobilization maneuvers). We meticulously research the correct plan of detachment of the cyst, which allows us to cause very limited bleeding, and we carry out a detachment (no stripping !!) with mono or bipolar coagulation of the vascular connections as close as possible to the capsule of the cyst and not on the parenchyma ovarian. Once the endometrioma has been removed, we apply Arista HA on the cystic bed by means of a light and flexible disposable spray device that allows to apply the hemospheres in a precise and direct way (Pict. 1,2). 


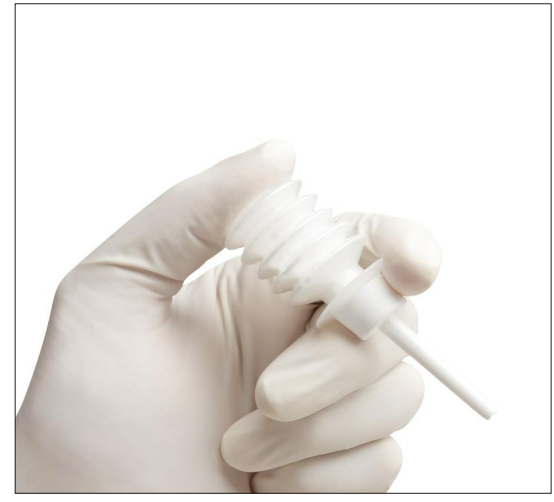

Picture 1

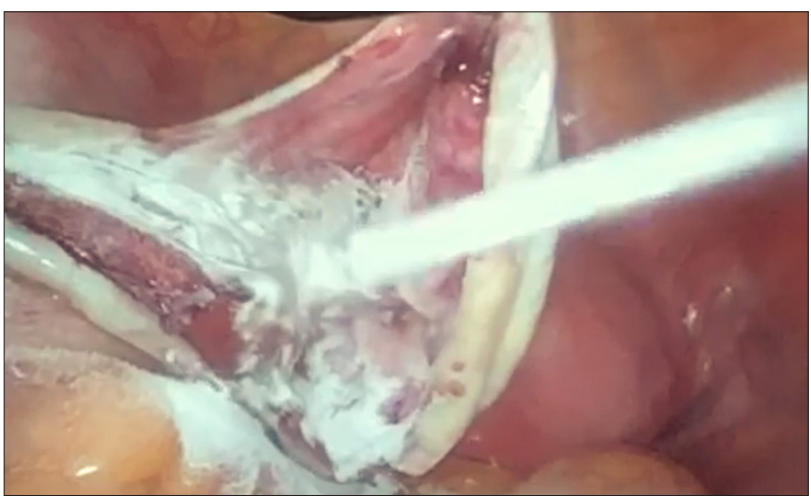

Picture 2

The hemostatic control is very often satisfactory: very rarely a minimal bipolar coagulation is performed, targeted by watertest. Transcutaneous ovarian suspension follows (ovaries must be placed outside the pelvis for 48 hours). Finally, we perform peritonectomy of the area of adhesion of the endometrioma to the pelvis and excision of all areas suspected for the presence of endometriotic foci. Finally we wash the pelvis with $250 \mathrm{ml}$ of Lactated Ringer's solution. Another $250 \mathrm{ml}$ is left in the pelvic cavity to avoid adhesions thanks to the ballotation of the intestinal loops.

Between January 2020 and July 2021 we performed laparoscopic surgery on 32 women with ovarian endometriosis: In 25 cases the endometrioma was unilateral and in 7 cases it was bilateral. The diameter of the endometriomas was between 4.5 and $8 \mathrm{~cm}$.

We performed the count of antral follicles (AFC) in all cases but sometimes we did not have an exact data, especially when the diameter of the endometrioma was greater than $5 \mathrm{~cm}$ and when the cyst was adherent to the pelvic wall in relation also to the association with extra-ovaric endometriosis.

In 20 cases the predominant symptom was dysmenorrhea, in 12 cases dysmenorrhea was associated with dyspareunia. Ovulatory pain and chronic pelvic pain were present in 24 women. Two patients were asymptomatic. Infertility was found in 18 patients.

We found peritoneal implants on the Douglas pouch in 20 women and on the bladder-uterine fold in 11 cases. In 13 cases we found the salpinges involvement and in 12 cases we removed nodules in the utero-sacral ligaments and / or in the round ones. In 5 women there is an association of DIE in the anterior or posterior compart.

We performed hemostasis with Arista $\mathrm{AH}$ powder in 27 patients.

In no case were there any post-surgical complications due to a defect in hemostasis.

48 hours after surgery we discharge the patients, after removing the wires of the ovarian suspension, with anti-inflammatory therapy for 10 or 15 days and, for women who do not wish to immediately seek a pregnancy, hormonal therapy (progestogens or estrogen-progestins) for at least 6 months.

Three months from surgery we perform the trans-vaginal ultrasound for Antral Follicle Count on the operated ovary. We perform AFC on both ovaries only in women with immediate pregnancy desire to assess the need for referral to PMA centers.

We know that Antral Follicle Count and Anti-Mullerian Hormone are parameters that are generally used in the prediction of ovarian functional reserve, but for our evaluation we have chosen the Antral Follicle Count. We have also confirmed the correctness of our choice from the study published by Inal in 2019 which concludes by claiming that no perfect marker exists in the evaluation of ovarian reserve and response, but which strongly suggests that AFC is a better reflection of ovarian response than $\mathrm{AMH}$ in women with endometrioma, even for those who undergoing an in vitro fertilization [21].

The evaluation of the AFC after 3 months from the intervention (in 24 women with complete follow-up) gave the following results: in patients with unilateral endometrioma the AFC was between 5 and 8 (mean value 6.3), in patients with bilateral endometriosis AFC ranged from 5 to 7 (mean value 5.8).

\section{Conclusions}

It is extremely necessary to reiterate the concept that it is the task of the gynecologist, who surgically tackles ovarian endometrioma, not only to try to remove the disease but also, and above all, to safeguard the fertile capacity of the woman. From this point of view, even small precautions must be clearly present in the baggage of the surgical technique. We believe it is important to remove the areas of the peritoneum when the endometrioma adheres to the pelvic wall to eradicate the disease and we believe it is important to have subsequent extra-pelvic ovarianpexy for 48 hours so that the peritoneum is reconstituted by budding. The control of haemostasis is very important and sometimes difficult, especially when approaching the ovarian hilum or when an adequate neckline plan is not respected during stripping (which must be as soft as possible because it often removes a good number of follicles healthy). In these cases, the use of coagulation can be effective but irreversibly damages the healthy ovarian parenchyma. The hemostatic suture is effective but is sometimes difficult to perform and can cause local ischemia. With these two haemostatic techniques there is a risk of further damaging the follicular reserve already altered by endometriosis. The use of Arista $\mathrm{AH}$ powder allows a 
highly effective and easy to use haemostasis that fully respects the residual ovarian parenchyma after stripping. It also reduces the operating time, since its application is very rapid, and blood losses, also reducing perioperative complications.

At the end, we like to underline that the good values resulting from the AFC control confirm that the surgical technique we use for ovarian endometrioma removal is particularly respectful of the ovarian follicular reserve and that therefore, even if it can be improved, it can be established as a preferential technique for treating this pathology.

\section{Acknowledgement}

The Authors declare that they have no conflict of interest with the manufacturer of Arista $\mathrm{AH}$ (Becton, Dickinson and Company - 1 Becton Drive - Franklin Lakes, NJ 07417-1880)

\section{References}

1. Nnoaham KE, Hummelshoj L, Webster P, Thomas d'Hooghe, Fiorenzo de Cicco Nardone, et al. (2011) World Endometriosis Research Foundation Global Study of Women's Health care. Impact of endometriosis on quality of life and work productivity: a multicenter study across ten countries. Fertil Steril 96: 366-373.

2. Matsuzaki S, Schubert B (2010) Oxidative stress status in normal ovarian cortex surrounding ovarian endometriosis. Fertil Steril 93: 2431-2432.

3. Vercellini P, Chapron C, De Giorgi O, Dario Consonni, Giada Frontino, et al. (2003) Coagulation or excision of ovarian endometriomas? Am J Obstet Gynecol 188: 606610.

4. Serdar E Bulun (2014) Ovarian Endometriosis: The Nemesis of Eggs. Fertil Steril 101: 938-939.

5. Jadoul P, Kitajima M, Donnez O, Jean Squifflet, Jacques Donnez, et al. (2012) Surgical treatment of ovarian endometriomas: state of the art? Fertil Steril 98: 556-563.

6. Kitajima M, Dolmans MM, Donnez O, Hideaki Masuzaki, Michelle Soares, et al. (2014) Enhanced follicular recruitment and atresia in cortex derived from ovaries with endometriomas - Fertil Steril 101: 1031-1037.

7. Nicolaus K, Bräuer D, Sczesny R, Jorge Jimenez-Cruz, Klaus Bühler, et al. (2019) Endometriosis reduces ovarian response in controlled ovarian hyperstimulation independent of AMH, AFC, and women's age measured by follicular output rate (FORT) and number of oocytes retrieved. Arch Gynecol Obstetr 300: 1759-1765.

8. Leone Roberti Maggiore U, Scala C, Venturini PL, Annalisa Abbiati, Guido Ragni, et al. (2015) Endometriotic ovarian cysts do not negatively affect the rate of spontaneous ovulation - Hum Reprod 30: 299-307.

9. Majedah Al-Azemi, Andrés Lopez Bernal, Jo Steele, I Gramsbergen, D Barlow, et al. (2000) Ovarian response to repeated controlled stimulation in in-vitro fertilization cycles in patients with ovarian endometriosis. Hum Reprod 15: 72-75.

10. Roman H, Tarta O, Pura I, Ioana Opris, Nicolas Bourdel, et al. (2010) Direct proportional relationship between endometrioma size and ovarian parenchyma inadvertently removed during cystectomy, and its implication on the management of enlarged endometriomas. Hum Reprod 25: 1428-1432.

11. Somigliana E, Benaglia L, Vigano' P, M Candiani, P Vercellini, et al. (2011) Surgical measures for endometriosis-related infertility: a plea for research. Placenta 32: 238-242.

12. Hurt RI, Hickey M, Maouris P (2008) Cochrane Reviews - Excisional surgery versus ablative surgery for ovarian endometriomata.

13. Chun-Hua Zhang, Ling Wu, Pei-Quan Li (2016) Clinical study of the impact on ovarian reserve by different hemostasis methods in laparoscopic cystectomy for ovarian endometrioma. Taiwanese Journal of Obstetrics \& Gynecology 55: 507-511.

14. Pergialiotis V, Prodromidoua A, Frountzasa M, Konstantinos Bitos 2, Despina Perrea, et al. (2015) The effect of bipolar lectrocoagulation during ovarian cystectomy on ovarian reserve: a systematic review. $\mathrm{Am} \mathrm{J}$ Obstet Gynecol 213: 620-628.

15. Asgari Z, Rouholamin S, Hosseini R, Mahdi Sepidarkish, Leila Hafizi, et al. (2016) Comparing ovarian reserve after laparoscopic excision of endometriotic cysts and hemostasis achieved either by bipolar coagulation or suturing: a randomized clinical trial. Arch Gynecol Obstet 293: $1015-1022$.

16. Mahmoud TH, El-Agwany AS (2016) Safety of hemostatic sutures for hemostasis of the ovarian bed after endometriotic ovarian cystectomy. Indian J Gynecol Oncol 14:36.

17. Takashima A, Takeshita N, Otaka K, Toshihiko Kinoshita. Effects of bipolar electrocoagulation versus suture after laparoscopic excision of ovarian endometrioma on the ovarian reserve and outcome of in vitro fertilization. $J$ Obstet Gynaecol Res 39: 1246-1252.

18. Ata B, Turkgeldi E, Seyhan A, Urman B (2015) Effect of hemostatic method on ovarian reserve following laparoscopic endometrioma excision; comparison of suture, hemostatic sealant, and bipolar dessication. A systematic review and meta-analysis. J Minim Invasive Gynecol 22: 363-372.

19. Choi C, Kim WY, Lee DH, San Hui Lee (2018) Usefulness of hemostatic sealants for minimizing ovarian damage during laparoscopic cystectomy for endometriosis. Gynecol Obstet Res 44: 532-539.

20. Bardi M, Pezzetti G, Rosaschino P, G Gangarossa, A Iliakis, et al. (2020) Ovarian Endometrioma Surgery How to safeguard the Follicular Reserve 5: 1-5.

21. Inal ZO, Engin Ustun Y, Yilmaz N (2019) Does the antiMüllerian hormone truly reflect ovarian response in women with endometrioma? J Obstet Gynaecol 39: 516521.

Copyright: (C2021 Massimo Bardi. This is an open-access article distributed under the terms of the Creative Commons Attribution License, which permits unrestricted use, distribution, and reproduction in anymedium, provided the original author and source are credited. 